\title{
HYDROGENATED AMORPHOUS SILICON DEPOSITED ON THE ASIHTEST CIRCUIT FOR RADIATION DETECTION
}

\author{
M. DESPEISSE ${ }^{1}$, G. ANELLI ${ }^{1}$, P. JARRON ${ }^{1}$, D. MORAES ${ }^{1}$, \\ A. NARDULLI ${ }^{2}$, N. WYRSCH ${ }^{3}$ \\ ${ }^{1}$ CERN, CH-1211 Geneva, Switzerland, \\ ${ }^{2}$ Institute for Particle Physics, ETH, CH-8093 Zurich, Switzerland \\ ${ }^{3}$ IMT, Rue A.-L. Breguet 2, CH-2000 Neuchatel, Switzerland
}

\begin{abstract}
Radiation detectors were developed by depositing $20 \mu \mathrm{m}$ thick hydrogenated amorphous silicon (a-Si:H) sensors directly on top of the so-called aSiHtest integrated circuit. This circuit was designed in a quarter micron CMOS technology as a global test circuit for this novel detector technology. The sensor leakage current turned out to be limiting the performance of the whole detector. Its detailed study is presented together with design techniques to reduce it. Direct detections of 10 to $50 \mathrm{keV}$ electrons were demonstrated on integrated thin strip structures with a pitch down to $10 \mu \mathrm{m}$. Results are shown to understand the potential of the technology for ionizing radiation detection.
\end{abstract}

\section{Thin Film on ASIC technology for radiation detection}

In addition to state-of-the-art silicon detectors, a number of innovations aimed at improving radiation detection speed, spatial resolution, radiation hardness or costs are being developed to greater maturity [1]. Their development is of primary interest for future high energy physics experiments and for biomedical imaging where alternative solutions to standard silicon detectors will be needed [2]. In this context, we studied the emerging Thin Film on ASIC (TFA) technology, which consist in the deposition of a hydrogenated amorphous silicon (a-Si:H) sensor directly on top of the readout integrated circuit [3]. a$\mathrm{Si}: \mathrm{H}$ is widely industrialized in thin films $(<1 \mu \mathrm{m})$ and it was shown to potentially offer radiation hard alternatives to silicon devices [4]. The vertical integration of the sensor on the electronic readout enables a level of integration comparable to monolithic pixel detectors while it still presents the advantages of the hybrid pixel approach as the sensing device and electronic readout can be designed, optimized and biased separately. However, sensors thicker than standard a-Si:H devices have to be deposited and fully depleted to provide adequate signal to noise ratio for particle detection. Sensors with a thickness above $50 \mu \mathrm{m}$ can be grown but would necessitate more than $1.1 \mathrm{kV}$ for full depletion and certainly be very hard to deplete [5]. Sensors with thickness up to 
about $30 \mu \mathrm{m}$ were thus deposited in our studies. They are thick for an a-Si:H device but thin compared to standard crystalline silicon detectors (which have thicknesses above $150 \mu \mathrm{m}$ ). Fewer charges are therefore expected to be created by the passage of a particle through a TFA detector than through a silicon detector, resulting in the most important limitation and challenge of the TFA technology. The development of this technology thus requires full depletion of the deposited a-Si:H sensors and the design of very low noise pre-amplifiers $(<$ $100 \mathrm{e}^{\mathrm{e}} \mathrm{rms}$ ) able to read out the small signal induced by a particle going through the thin a-Si:H sensor.

\section{The aSiHtest detector}

A TFA detector is built by consecutive depositions of n-doped, intrinsic and p-doped (n-i-p) a-Si:H films on top of the readout circuit (Fig. 1). Depositions were done by Plasma Enhanced Chemical Vapor Deposition at $200{ }^{\circ} \mathrm{C}$ at the Institute of Micro-technology of Neuchatel. The doped layers are deposited thinly $(\sim 30 \mathrm{~nm})$ because of a high defect density and the diode and i-layer thicknesses are thus equal. A transparent conductive oxide (TCO) is deposited on the a-Si:H sensor p-layer and defines the detector's common top electrode.

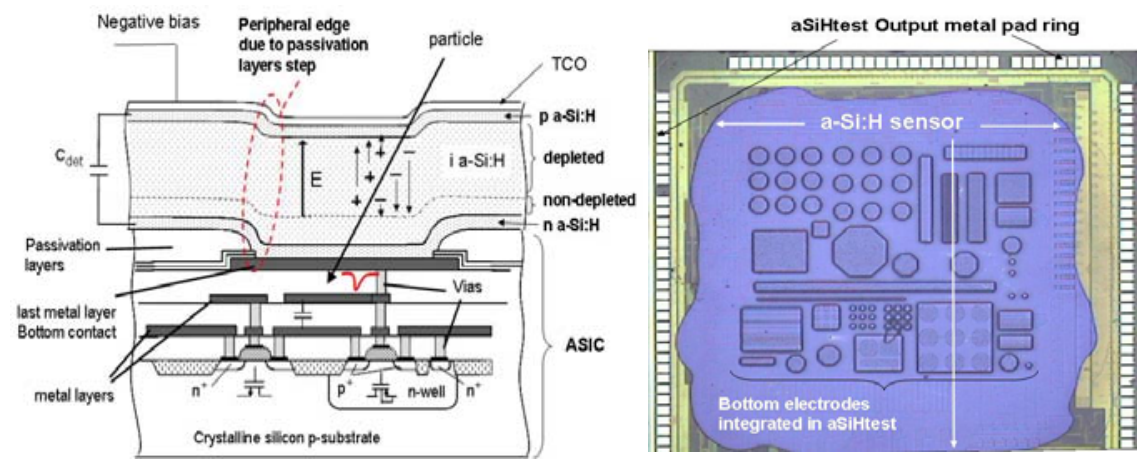

Fig. 1. Left: Schematic cross section of a TFA detector based on the deposition of n-i-p a-Si:H layers and TCO electrode on top of a CMOS circuit with 3 levels of metal. Right: A TFA detector made of a $20 \mu \mathrm{m}$ thick a-Si:H sensor and an aSiHtest circuit.

Sensors were first successfully deposited on the AFP and MACROPAD circuits and the developed TFA detectors are presented in [5]-[6]. The aSiHtest circuit was designed in a quarter micron CMOS technology as a global test chip to fully characterize the TFA technology. TFA detectors were built on this circuit with $20 \mu \mathrm{m}$ thick a-Si:H sensors (Fig. 1). In standard CMOS technologies, integrated circuits are protected with passivation layers which cover its surface. Openings in these layers are made to allow external access to metallic pads designed in the topmost metal layer, and in the case of TFA detectors to provide back contacts to the a-Si:H diodes. The metal pads and the openings define the 
detector segmentation, and openings are usually done inside the pads, the $5 \mu \mathrm{m}$ thick passivation layers inducing unevenness of the circuit (Fig. 1). The different metal structures integrated in the aSiHtest are designed with passivation layers opened either on their top (inside the pad) or outside the metal pad, in order to study the impact of the passivation layers step on detector performance. These different metal structures segment the TFA detector in either strips or pixels. They are either connected to current mirror stages which provide a selectable current gain of $10^{2}$ to $10^{5}$ to study the leakage current of the a-Si:H sensor or to active feedback pre-amplifiers to perform radiation detection. Integrated amplifiers exhibit either a 5 ns peaking time and an equivalent noise charge (ENC) of $160 \mathrm{e}^{-} \mathrm{rms}$ or a 25 ns peaking time for an ENC of $70 \mathrm{e}^{-}$rms (for a $1 \mathrm{pF}$ maximum input capacitance).

\section{Leakage current mechanisms}

The high reverse voltages needed to deplete the "thick" deposited a-Si:H sensors and the deposition on non-flat ASIC surface were shown to strongly increase the sensor's leakage current [5]-[6], enhancing mechanisms negligible in standard a-Si:H devices. These high currents prevent the full depletion of the deposited sensors, limiting the active volume of interaction and lowering the weighting field (and so the induced signal amplitude) as the non-depleted layer is non conductive. The understanding of the mechanisms governing the leakage current in TFA detectors and their reduction is thus of primary interest for the development of the technology. The evolution of the leakage current density of $20 \mu \mathrm{m}$ thick sensors deposited on aSiHtest circuit were measured on the different integrated structures as a function of the temperature and results are presented in Fig. 2. A current induced by thermal generation enhanced by electric field E through Poole-Frenkel mechanisms can be expressed as [7]:

$$
\mathrm{I}=\mathrm{I}_{0} \exp \left(-\mathrm{E}_{\mathrm{A}} / \mathrm{kT}\right) \quad(1) ; \quad \mathrm{E}_{\mathrm{A}}=\mathrm{E}_{\mathrm{G}} / 2-\gamma \sqrt{\mathrm{E}}
$$

$E_{G}$ is the material bandgap, $E_{A}$ is the activation energy, i.e. the energy needed to activate ionisable defects, $\gamma$ is the Poole-Frenkel constant, $\mathrm{I}_{0}$ the maximum current (for $\mathrm{E}_{\mathrm{A}}=0$ ), $\mathrm{T}$ the temperature and $\mathrm{k}$ the Boltzmann constant.

Experimental data presented in Fig. 2 is well fitted by equations (1) and (2), showing that the leakage current in the TFA detector can be attributed to PooleFrenkel reduction of the activation energy, enhancing the thermal generation of charges. The activation energy was extracted for different structures and it was shown to decrease for increasing applied reverse voltages. Values equal to $0.49 \mathrm{eV}$ and $0.42 \mathrm{eV}$ were measured for $80 \mathrm{~V}$ and $160 \mathrm{~V}$ biases respectively, 
while it is expected to be at about $0.9 \mathrm{eV}$ for no applied biases. Experimental results presented in Fig. 2 right show that reduction of the TFA detector dark current could then be obtained by opening the passivation layers outside the metal pads, i.e. by planarization of the circuit surface. Standard openings in the passivation layers inside the metal pad lead to a non-planar profile of the a-Si:H sensor on top of the metal pad, inducing high electric field zones at the pixel periphery and thus increasing leakage currents. Significant reduction could also be obtained by a planarization process of the ASIC surface.
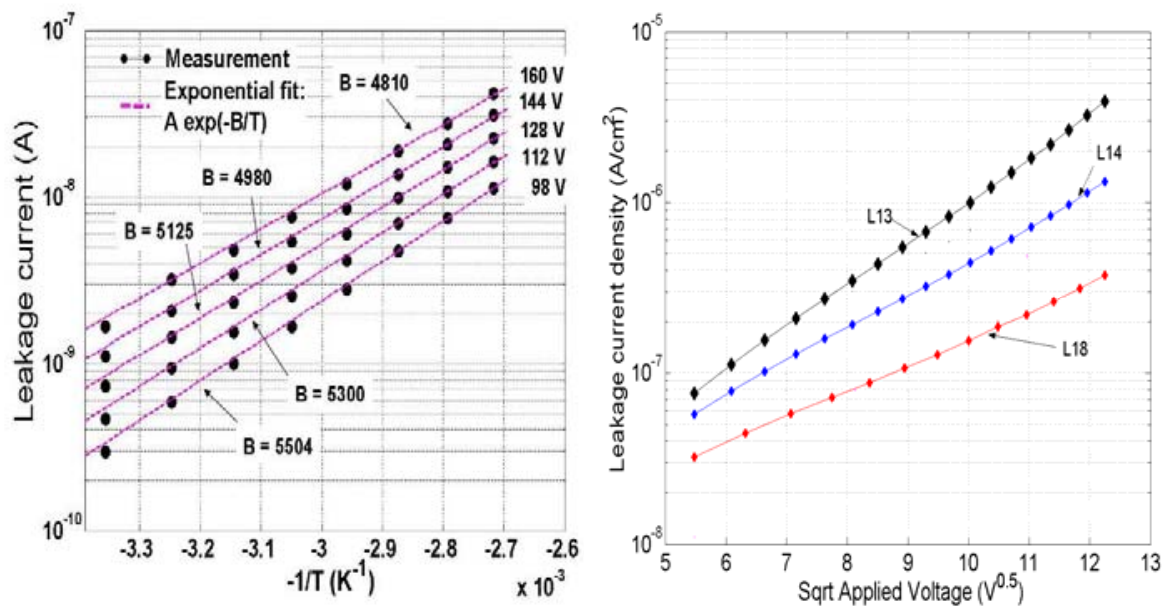

Fig. 2. of leakage current measured on a $20 \mu \mathrm{m}$ thick a-Si:H sensor on top of an aSiHtest circuit Left: Evolution with temperature. Right: for pixel pads with openings inside the metal pad (L13), at $6 \mu \mathrm{m}$ outside the metal pad (L14) and at $40 \mu \mathrm{m}$ outside the metal pad (L18).

\section{Radiation detection}

Direct detections of 10 to $50 \mathrm{keV}$ electrons were also carried out on the aSiHtest detectors. Results obtained on high resolution strip structures, $6.6 \mu \mathrm{m}$ wide and separated by $4 \mu \mathrm{m}$ to $13.4 \mu \mathrm{m}$, each connected to one of the implemented lownoise pre-amplifiers, are presented in Fig. 3. Most of electrons inducing a signal on the strip S2 do not induce a significant signal on the neighboring strips. Few signals seen on $\mathrm{S} 2$ induce a low signal on $\mathrm{S} 1$ (which is at $4 \mu \mathrm{m}$ ), but these events are rare $(<20 \%)$ and can be explained by the tortuous path of few $\mathrm{keV}$ electrons in matter. Low charge sharing can therefore be considered on thin strip structures on the developed TFA detectors. This can be explained by the low mobility in a-Si:H and thus the low diffusion coefficient of about $10^{-2} \mathrm{~cm}^{2} / \mathrm{s}$ for electrons and $10^{-4} \mathrm{~cm}^{2} / \mathrm{s}$ for holes [8]. However, charges drift under very high electric fields on short distances (thin sensor of $20 \mu \mathrm{m}$, maximum drift time of electrons of about $18 \mathrm{~ns}$ [5]) so that lateral diffusion of a charge packet will be 
small. A deviation perpendicular to the electric field of less than $1 \mu \mathrm{m}$ rms for a charge packet created at the p-i interface in a $20 \mu \mathrm{m}$ thick sensor can be expected. These results thus show a very interesting potential of the TFA technology for the detection of low energy electrons and for the construction of a high spatial resolution detector.

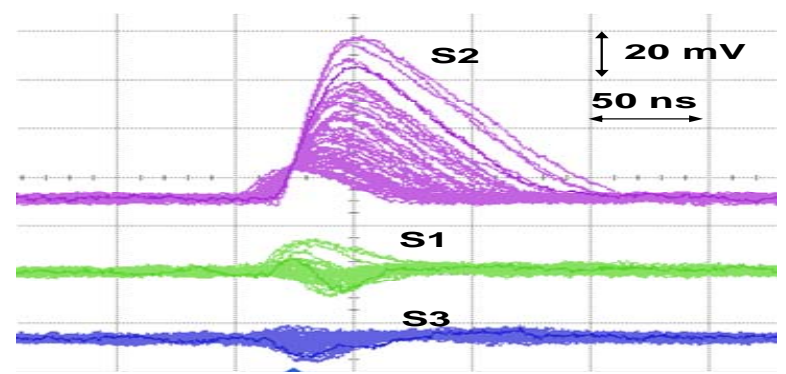

Fig 14. Signals created by 10 to $50 \mathrm{keV}$ electrons on a $20 \mu \mathrm{m}$ thick a-Si:H sensor deposited on aSiHtest circuit. The circuit is reverse biased with $240 \mathrm{~V}$. Top: readout is done on 3 neighboring strips, S1, S2 and S3, trigger on S2. Bottom: 100 superimposed signals created by electrons from the source.

\section{$\underline{\text { CONCLUSION }}$}

$\mathrm{N}$-i-p a-Si:H sensors with thicknesses up to $20 \mu \mathrm{m}$ were successfully deposited on top of aSiHtest integrated circuits. Edge effects due to the non-flatness of the circuits were demonstrated to increase the sensor leakage current, limiting the detector performance, but specific ASIC design of metal pad was shown to permit to lower these currents. Finally, direct detection of ionizing particles was performed on both pixels and strips structures, demonstrating an interesting potential of the technology for high spatial resolution detection.

\section{REFERENCES}

[1] N. Wermes. Nucl. Instr. And Meth. In Phys. Res. A 541 (2005), 150-165.

[2] M. Moll, et al. Nucl. Instr. And Meth. In Phys. Res. A 546 (2005), 99-107.

[3] P. Jarron, G. Anelli, S. Commichau, M. Despeisse, G. Dissertori, C. Miazza, D. Moraes, A. Shah, G. Viertel, N. Wyrsch, Nucl. Instr. And Meth. In Phys. Res. A518 (2004) pp. 366-372.

[4] J.R. Srour, G. J. Vendura, D. H. Lo, C.M.C. Toporow, M. Dooley, R.P. Nakano and E.E. King, IEEE Trans. On Nucl. Sc., vol. 45, no. 6, (1998), pp. 2624-2630.

[5] M. Despeisse et al. Nucl. Instr. And Meth. In Phys. Res. A518 (2004), pp. 357-361

[6] D. Moraes et al., J. Non-Cryst. Solids 338-340, 2004, pp. 729-731.

[7] J. B. Chévrier and B. Equer, J. Appl. Phys. 76 (11), 1994, pp. 7415-7422.

[8] Q. Gu, et al., Phys. Rev. Lett., Vol. 76, 1996, pp. 3196-3199. 